\title{
Symposium
}

\section{Kapital, Ideologie und Gesellschaftsstruktur}

Symposiumsbeitrag zu: Thomas Piketty, Kapital und Ideologie. München: C.H. Beck 2020, 1312 S., gb., 39,95€

Besprochen von Prof. Dr. Bernd Baldus: Department of Sociology, University of Toronto, E-Mail: bernd.baldus@utoronto.ca

https://doi.org/10.1515/srsr-2021-0002

Schlüsselwörter: Soziale Ungleichheit, Ideologie, Sozialgeschichte

Das Hauptargument von Piketty’s „Kapital im 21. Jahrhundert“, dem Vorgänger des hier besprochenen Buches, war, dass in kapitalistischen Gesellschaften das Wachstum des Profits aus der wirtschaftlichen Nutzung von Kapital tendenziell das Wachstum des Brutto-Inlandsprodukts übertrifft. Die Akkumulation vererbten Kapitals ist eine treibende Kraft dieses Prozesses und führt zu einer ständig größeren Konzentration von Reichtum und einem zunehmenden Einfluss der Kapitalbesitzer auf Sozial- und Wirtschaftspolitik. Diese These war prägnant und einfach, und Piketty belegte sie durch detaillierte Verteilungsstatistiken. Sein Argument revidierte Marx’ These von der sinkenden Profitrate, präsentierte sich jedoch als eine Gesetzmäßigkeit, die nur gelegentlich durch soziale Umwälzungen oder politische Eingriffe modifiziert werden konnte. Er entfernte sich daher nicht allzu weit von Marx' nie völlig gelöstem Widerspruch zwischen historischem Gesetz und menschlicher Autonomie.

Piketty's neues Buches will auf zwei Kritiken von „Kapital im 21 Jahrhundert“ antworten: seine geographische Begrenzung auf Verteilungsentwicklungen in Europa und den USA und die Vernachlässigung von „politisch-ideologischen Entwicklungen, von denen die Entwicklung der Ungleichheit und Umverteilung sich nicht ablösen lässt“ (Piketty: 34). Man könnte noch eine dritte Kritik hinzufügen: Piketty beschrieb die Evolution von Ungleichheit als einen weitgehend determinierten Prozess, der, sobald bestimmte Voraussetzungen eintraten, einen mechanischen, voraussagbaren Verlauf nahm.

Um es gleich zu sagen, scheint mir, dass es Piketty besser gelungen ist, den ersten als die beiden anderen Vorwürfe zu entkräften. Sein Buch, mit 1281 Seiten Text fast doppelt so lang wie sein Vorgänger, beginnt mit einer auf den gegenwärtigen Stand gebrachten Darstellung der Verteilungsentwicklung in einzelnen 
Ländern und Regionen der Welt. Piketty zeigt sowohl indigene Strukturänderungen wie auch die oft langdauernden Auswirkungen europäischer Kolonialpolitik auf soziale Ungleichheit in abhängigen Ländern. Wer jedoch hofft, wie der Titel des Buches verspricht, mehr über die Rolle von Ideologie bei der Verfestigung und Erhaltung sozialer Ungleichheit zu lernen, wird Piketty's Ideologiebegriff zu begrenzt finden.

Das Buch ist leicht lesbar und enthält eine Vielzahl von langfristigen und vergleichenden Grafiken, die über Zeiträume von 1380 bis zur Gegenwart ein außerordentlich detailliertes und instruktives Bild historischer Verteilungsentwicklungen geben. Piketty teilt diese in fünf Phasen: Frühe dreigliedrige Eigentumsstrukturen in Europa und anderen Ländern, neue proprietaristische Eigentumsideen in der französischen Revolution, die Konsolidierung nachrevolutionärer Ungleichheit im 19. Jahrhundert, ihr Zerfall zwischen 1914 und 1945 und eine Periode relativer Gleichheit zwischen 1950 und 1980 und schließlich das erneute hyperkapitalistische Wachstum der Ungleichheit nach 1980. Der Zusammenhang zwischen strukturellen Änderungen und den damit verbundenen Eigentums- und Verteilungsideologien geht allerdings in der Vielfalt von Details und der Länge des Buchs oft verloren. Ich möchte deshalb den Inhalt kurz zusammenfassen, nicht zuletzt um es leichter zu machen, einzelne Teile des Buches zu lesen, was durchaus lohnend und interessant sein kann. Das Buch hat zwar kein Sachverzeichnis, aber ein Personenregister, ein Verzeichnis von Grafiken und Kapiteln, sowie eine Einleitung, die ein Herausgreifen bestimmter Themen oder Länder vereinfachen.

Piketty beginnt mit einer Analyse der trifunktionalen ideologischen Rechtfertigung der Besitz- und Machtverhältnisses zwischen Bauern, Adel und Klerus im europäischen Feudalismus. Ähnliche Strukturen findet er auch in Indien, China, Japan und islamischen Gesellschaften. Ideologisch verstanden sie sich als Systeme gegenseitiger Verpflichtungen in denen eine Klasse, bei weitem die größte, die Nahrungsgrundlagen für alle drei Gruppen sicherstellte, während eine zweite für militärischen Schutz und die dritte für religiöse Fürsorge sorgte. Parallel dazu verliefen feste Eigentumsregelungen, die eng an diese Positionen gebunden waren. Ein Übergang von der unteren in die nächsthöheren Klassen war praktisch unmöglich.

Die Französische Revolution brach dieses dreigliedrige System auf, aber nur teilweise: Klerus und Kirche verloren fast ihr gesamtes Eigentum, und der Grundbesitz des Adels wurde halbiert. Die grundlegende ideologische Idee, Eigentum als Garant gesellschaftlicher Stabilität, blieb jedoch erhalten. Die Hauptneuerungen der Revolution bestanden einerseits im Transfer „unverdienter“ rechtlicher Privilegien von Adel und Klerus auf den Zentralstaat. Andererseits wurde die alte trifunktionale Eigentumsideologie durch eine neue, proprietaristische ersetzt, die Gleichheit als das Recht des einzelnen Bürgers definierte, entsprechend seiner 
Fähigkeiten, Reichtum zu erwerben und zu nutzen und „damit auf natürlichem Wege zum Wohlstand aller und zu sozialer Harmonie zu führen“ (Piketty: 165). Damit wurde die Frage einer gerechten Verteilung und die darin verborgene Gefahr einer zu weitgehenden Umverteilung sorgfältig umgangen. Condorcet und Paine hatten bereits für ein progressives Steuersystem und ein allgemeines Grundeinkommen plädiert. Solche Forderungen ließen schnell Befürchtungen über die Folgen einer unkontrollierten Ausdehnung des Gleichheitsbegriffes aufkommen. Die proprietaristische Eigentumsideologie wurde deshalb erfinderischer. Um die grundlegende Unverletzlichkeit des Eigentums rankte sich nun eine Vielzahl von Rechtfertigungen, von der Verbindung von Reichtum mit persönlicher Leistung oder ererbter Fähigkeit bis zur ideologischen Konstruktion von Ungleichheit als Vorbedingung für die Zivilisierung der unteren Klassen. Hier zeigten sich auch die ersten ideologischen Umrisse moderner Politik, wo konservative Parteien in jeder Umverteilung eine Bedrohung der gesellschaftlichen Ordnung sehen, während sozialdemokratische Parteien Umverteilung befürworten, das Prinzip Eigentum aber nicht in Frage stellen.

Diese neuen proprietaristischen Ideologien begünstigten einen raschen Anstieg der Ungleichheit im 19. Jahrhundert. Die Restauration in Frankreich und das rapide Wachstum industrieller Profite führte $\mathrm{zu}$ einer hohen Konzentration von privatem Reichtum in den Händen neuer Kapitalbesitzer. Dazu kamen enorme Einnahmen aus der Ausbeutung von Sklaverei und Kolonien, die auch nach dem Ende der Sklaverei weiterbestanden, da Sklavenhalter für den Verlust des ,Marktwertes' ihrer Sklaven entschädigt wurden. Piketty folgt der Pomeranz-These, dass die stagnierende Entwicklung europäischer Kolonien nicht aus ihrer kulturellen Rückständigkeit resultierte, sondern aus der militärischen und wirtschaftlichen Überlegenheit, die sich kurzfristig aus der Rivalität europäischer Mächte und der Ausbeutung ihrer Kolonien ergab und deren mögliche selbstständige Entwicklung verhinderte.

Die hohe Eigentumskonzentration in den USA und Europa Ende des 19. und anfangs des 20. Jahrhunderts endete nach 1914. Der Zusammenbruch des europäischen Kolonialsystems, erstarkende sozialdemokratische und kommunistische Parteien und der wachsende wirtschaftliche und militärische Wettbewerb zwischen europäischen Nationalstaaten und der daraus folgende Weltkrieg führten zu einer tiefgreifenden ideologischen Transformation und einer Periode relativer sozialer Gleichheit, die bis etwa 1980 dauerte. Gleichzeitig sah diese Zeit hohe wirtschaftliche Wachstumsraten, für Piketty ein Beweis, dass eine Reduzierung der Ungleichheit keineswegs auf Kosten der Wirtschaftsleistung gehen muss. Allerdings hatte dieses „goldene Zeitalter der Sozialdemokratie“ (Piketty: 612) Schwächen. Umverteilung, öffentliches Eigentum und Mitbestimmung wurden nur in wenigen Ländern wie Schweden und Deutschland, und auch dort nur unvollkommen, erprobt. 
Die weitreichendste Umverteilung unternahmen in dieser Periode kommunistische Regierungen in der Sowjetunion und China. Das Ziel der Russischen Revolution war, Privateigentum weitmöglichst abzuschaffen. Eigentum bestand nun aus ,sozialistischem Eigentum‘ (verstaatlichte Unternehmen und kollektive Landwirtschaft), ,persönlichem Eigentum‘ (Gegenstände des täglichen Verbrauchs), und ,Privateigentum', das strafbar machte und zu hohen Inhaftierungsquoten führte. Dazu kam die Zentralisierung des Verbrauchs aufgrund der Annahme, dass menschliche Bedürfnisse homogen und voraussagbar seien. Diese Begrenzung geriet in ständigen Widerspruch zu der Vielfalt und Wandelbarkeit individueller Interessen. Obgleich die sowjetische Wirtschaft bis 1990 auf etwa 60\% des europäischen Lebensstandards aufschloss, litt das System unter ständiger Angst, zu viel Privatisierung von Besitz und Verbrauch zuzulassen. Nach dem Zerfall der Sowjetunion stieg die Ungleichheit in Russland schnell auf ein selbst die USA übertreffendes Niveau. In China wurde die zunächst radikale Kollektivierung nach den negativen wirtschaftlichen Folgen der Kulturrevolution von einem gemischten System ersetzt. Staatseigentum an Unternehmen wurde zwischen 1978 und 2018 halbiert. Gleichzeitig nahm die Ungleichheit in der chinesischen Bevölkerung erheblich zu. Der ,kommunistische“ Charakter des Systems verlagerte sich auf eine Zentralisierung der politischen Macht, die sich als Repräsentant des Volkswillens verstand und ein umfassendes Überwachungssystem aufbaute.

Piketty sieht die letzte Phase der Entwicklung von Ideologie und Kapital in einem Hyperkapitalismus, der ideologisch von neoliberalen Ideen der Marktfreiheit und dem Rückzug des Staates aus der Wirtschaft gekennzeichnet war. Als Folge nahm die Ungleichheit nach 1980 sowohl innerhalb wie auch zwischen verschiedenen Ländern drastisch zu. Hohe Einkommen kamen zunehmend aus Finanztransaktionen statt aus industrieller Produktion, und aus Steuerflucht und undurchsichtigen Finanzgeschäften. Staaten zogen sich aus der statistischen Erfassung von Einkommens- und Vermögensverteilung zurück. Dies galt auch für die Berechnung der Kosten des Klimawandels. CO2 Emissionen wurden nur unzureichend erfasst, und ihre Besteuerung blieb begrenzt und stark regressiv, da sie als fester Steuersatz vor allem den allgemeinen Verbrauch traf, statt hohe Emissionen höher zu besteuern. Dagegen nahm die Rolle des Staates bei der Geldschöpfung zu. Das ,quantitative easing“ der Notenbanken machte einen Kapitalismus auf Pump möglich und rettete marode Unternehmen und Banken wie in der Finanzkrise von 2008.

Diese Veränderungen spiegelten sich auch im Wahlverhalten. Zwischen 1950 und 1980 folgte es in den USA, England und Frankreich im Allgemeinen der Klassenstruktur: Wähler mit niedrigem Einkommen zogen linke Parteien vor, während obere Klassen konservative Parteien bevorzugten. Seit etwa 1990 bevorzugten unterschiedliche intellektuelle und kulturbestimmende 'Eliten' und Wähler 
mit hohen Einkommen wechselnde Parteien und Koalitionen, während die Wahlbeteiligung der unteren Klassen zurückging.

Im letzten Teil seines Buches macht Piketty eine Reihe von konkreten Vorschlägen für eine Reduktion der Ungleichheit und einen partizipativen Sozialismus, der eine sozial gerechte Verteilungsordnung garantieren könnte. Dabei folgt er Rawls' Gerechtigkeitsprinzip: Ungleichheit ist akzeptierbar, solange sie die Lebensbedingungen der am wenigsten Begünstigten verbessert. Konkreter formuliert darf „das Ausmaß der Konzentration des Privateigentums und der daraus resultierenden Macht [...] das unbedingt erforderliche nicht übersteigen - Mittel, um das zu erreichen, sind insbesondere die stark progressive Eigentumssteuer, eine allgemeine Kapitalausstattung und die gleichmäßige Aufteilung von Mitspracherechten zwischen Beschäftigten und Aktionären“ (Piketty: 746).

Einmal geht es Piketty hier um ein rechtliches System, das die einseitige Macht der Eigentümer industriellen Kapitals reduziert. Das Problem bestehender Mitbestimmungsgesetze, wo bei Stimmengleichheit die Aktionäre ausschlaggebend sind, soll in größeren Unternehmen durch ein Höchststimmrecht von Großaktionären begrenzt werden. Darüber hinaus weist Piketty auf Universitäten, Stiftungen und Genossenschaften hin, die selbstverwaltende Alternativen zu hierarchischen kapitalistischen Organisationsformen zeigen.

Der zweite Pfeiler für eine gerechtere Verteilung ist eine Reform des Steuerwesens und die Benutzung der dadurch erzielten Erträge für eine breitere Streuung des Vermögens. Dazu schlägt Piketty progressive Einkommens-, Erbschaftsund Eigentumssteuern vor, die Sozial- und ökologische Ausgaben finanzieren, darunter ein Mindesteinkommen für ärmere Bevölkerungsgruppen und ein einmaliges Startkapital für junge Bürger. Als Rahmenbedingungen fordert er eine umfassende Steuer- und Finanztransparenz sowie ein in der Verfassung verankertes Prinzip der Steuergerechtigkeit.

Kapital und Ideologie ist ein langes und informationsreiches Buch. Zwei Vorzüge verdienen besonders hervorgehoben zu werden. Erstens konzentriert sich Piketty ausschließlich auf das Thema materieller Ungleichheit, ihrer historischen Trends und ihrer vergleichenden Entwicklung. Mir ist kein Äquivalent in der Ungleichheitsliteratur bekannt, das der analytischen Breite von Piketty`s Buch nahekommt. Aber es ist nicht nur der Reichtum an Information, sondern die konsequente Verfolgung des Themas selbst, welches das Buch zu einem bedeutenden Beitrag zur Verteilungsdiskussion macht. Einkommens- und Vermögensungleichheit sind aus der nordamerikanischen soziologischen Literatur weitgehend verschwunden, verdrängt von Studien ethnischer, sexueller und ,rassischer` Differenzen, deren Objektivität oft fragwürdig ist. Piketty will zeigen, dass Verteilungsentscheidungen weitreichende und langfristige gesellschaftliche Konsequenzen haben. 
Piketty's zweites Verdienst ist, eine deterministische Lesart der historischen Evolution von Ungleichheitsstrukturen zurückzuweisen. Dies mag eine Korrektur der linearen Argumentation von „Kapital im 21. Jahrhundert“ sein, aber sie ist wichtig. Wenn es so etwas wie eine Erbsünde der Sozialwissenschaften gibt, ist es die Annahme, dass Ungleichheit ein Produkt biologischer Auswahl, sozialer Gesetze oder menschlicher Rationalität ist. Der Ursprung dieser Idee lag in den schnellen technologischen und wissenschaftlichen Fortschritten des 19. Jahrhunderts. Sie weckten bei frühen Sozialwissenschaften die Hoffnung, auch für Gesellschaften Gesetzlichkeiten zu finden, die den Ablauf der Geschichte bestimmten. Dieser Gedanke schien Lösungen zu versprechen für den schnellen strukturellen Wandel und die sozialen Spannungen und Konflikte zwischen einer aufstrebenden Arbeiterschaft einerseits, und Kapitalbesitzern, deren soziale Position ideologisch noch nicht abgesichert war. Konservative Sozialwissenschaftler erklärten Ungleichheit als einen notwendigen Teil eines vorherbestimmten Entwicklungsweges, der in allgemeiner Prosperität endete. Kritiker wie Marx wiederum versprachen, dass soziale Gesetze den Kapitalismus mit einer gerechteren und gleicheren Gesellschaft ersetzen würden. Diese Illusion legte die Grundlage für positivistische und rationalistische Sozialwissenschaften des 20. Jahrhunderts. Zufall, irrationales Handeln, unvorhergesehene Resultate und alternative Entwicklungsmöglichkeiten verschwanden aus ihrem Vokabular.

Piketty hält es dagegen für wichtig, Kreuzungspunkte und mögliche Weggabelungen der menschlichen Geschichte zu untersuchen. „Zu behaupten, all diese Episoden (der Ungleichheitsentwicklung) [...] vom 18. bis zum 20. Jahrhundert [...] seien notwendig und unverzichtbar gewesen, um dem menschlichen Fortschritt zum Sieg zu verhelfen, hätte kaum Sinn. Es waren andere Wegverläufe und Ungleichheitsregime denkbar, und andere Wegverläufe und egalitäre Regime sind auch weiterhin möglich“ (Piketty: 163, 39). Piketty zeigt, dass progressive Einkommens- und Erbschaftssteuern, das allgemeine Wahlrecht, der Aufstieg sozialdemokratischer Parteien und die Abnahme der Ungleichheit nach dem ersten Weltkrieg sehr schnell und aus einer Vielzahl von Gründen eintraten.

Trotzdem geistern hier und da deterministische Gedanken durch das Buch. Hinter der feudalen trifunktionalen Rechtfertigung der Ungleichheit zwischen Bauern, Klerus und Feudalherren sieht Piketty eine allgemeine Logik, die ein Gleichgewicht zwischen arbeitenden, gebildeten und vermögenden gesellschaftlichen Elementen in vielen Gesellschaften garantiert (Piketty: 500, 602). Ungleichheit kann zu- oder abnehmen, unterstützt oder bekämpft werden, bleibt jedoch ein permanentes Merkmal menschlicher Gesellschaft, weshalb „[...] große, dauerhafte und weitgehend willkürliche Ungleichverteilungen von der Frage nach deren gewaltsamer oder unrechtmäßiger Herkunft sich immer wieder zu erholen neigen, sowohl in den modernen hyperkapitalistischen Gesellschaften als auch 
in alten Gesellschaften“ (Piketty:169). Wenn dem aber so ist, scheinen die Chancen gering, dass „egalitäre Regime auch weiterhin möglich“ sind.

Diese Passage wirft zwei Fragen auf, die Piketty auch in „Kapital im 21. Jahrhundert“ nicht oder nur unvollkommen beantwortet. Die erste ist, wie Ungleichheit überhaupt beginnt, gleichgültig, ob in egalitären frühen Gesellschaften oder im Übergang von relativer Gleichheit nach dem ersten Weltkrieg zum Hyperkapitalismus. Ich habe dieses Problem an anderer Stelle eingehender behandelt (Baldus, 2017). Die vereinfachte Antwort ist, dass, wie Darwin bereits erkannte, Kooperation ein elementarer Bestandteil menschlicher Evolution ist, in den frühesten Formen menschlicher Zusammenarbeit wie in moderner Forschung oder industrieller Produktion. Kooperation erfordert Vertrauen. Vertrauen schafft jedoch auch Gelegenheiten zur Ausnutzung der Zusammenarbeit anderer und zum Entstehen von Ungleichheit, die sich als Kooperation maskiert. Kooperation erlaubt uns, ein Vielfaches von dem zu erreichen, was uns als Einzelnen möglich ist, führt aber gleichzeitig zu komplexen Dynamiken, die unvoraussagbar Gleichheit oder Ungleichheit verursachen können. In welche Richtung solche Prozesse gehen, kann von einer Vielzahl auslösender Ereignisse abhängen.

Die zweite Frage ist, warum, wie Piketty richtig sieht, selbst gewaltsame und unrechtmässige Ungleichheitssysteme sich langfristig verfestigen können. Für ihn funktionieren Ideologien im wesentlichem als explizite Vorstellungen von Eigentum, konzipiert von seinen Besitzern, Kolonialpolitikern oder intellektuellen Zuträgern wie Calhoun oder Hayek. Diese werden dann popularisiert, um der breiten Bevölkerung zu suggerieren, dass „die Konkurrenz aller gegen alle es erfordere, den mobilsten Steuerzahlern immer größere Steuersenkungen einzuräumen“ und sie als Wohltäter zu bewundern, denen man so viele Neuerungen und Segnungen verdankt (Piketty: 1091). Moderne Ideologien wie Popularismus, Sozialnativismus, Nationalismus oder antiwissenschaftliche und antiglobale Ideologien scheinen aus Piketty's Sicht von oben nach unten in die breite Bevölkerung durchzusickern und sich dort mehr oder weniger selbstverständlich festzusetzen.

Solche gelenkten Prozesse sind ein wichtiger Teil der Ideologiegenese, aber nur ein Teil. Warum entstehen Ideologien so regelmäßig mit oder kurz nach dem Auftreten von Ungleichheit? Wie erklärt sich ihre konsolidierende Kraft, selbst wenn sie erkennbar „verrückt“ sind (Piketty: 51)? Piketty’s Antworten bleiben vage. Menschliche Geschichte „ist ihrer Struktur nach eine Geschichte der Ideologien, die (Gesellschaften) entwickeln, um ihre sozialen Gruppenbeziehungen, Eigentumsordnungen und Grenzregime durch ein komplexes und wandelbares Gefüge von Einrichtungen zu organisieren“ (Piketty: 1185). Ideologien erscheinen als ein quasi-naturwüchsiges, unüberwindbares „Bedürfnis menschlicher Gesellschaften, ihren Ungleichheiten einen Sinn zu geben, mitunter auch jenseits aller Vernunft“ (Piketty: 49, 50). Wie die Ungleichheit selbst, entstehen sie in „sämtli- 
chen menschlichen Gesellschaften” (Piketty: 342), oft, weil der Zusammenbruch eines Ungleichheitssystems wie des Feudalismus eine „Angst vor der Leere“ (168) hinterlässt, die von neuen Ideologien gefüllt werden muss.

In Wirklichkeit ist die Verfestigung von Ungleichheitsstrukturen das Resultat eines viel breiteren und außerordentlich komplexen Prozesses sozialer Kontrolle (Baldus, 2017). Diejenigen, die unter Ungleichheit leiden, erfinden oft ihren eigenen Weg, ihre Lage erträglicher zu machen, womit sie gleichzeitig aber auch unbeabsichtigt zur Erhaltung der Ungleichheit beitragen. Besitzer von Reichtum und Macht können Situationen zu ihrem Vorteil nutzen, an deren Entstehen sie nicht beteiligt waren. Marx schrieb im 18. Brumaire, dass der Klassenkampf in Frankreich Umstände und Beziehungen schaffte, die es Louis Bonaparte III., einem Clown von „groteskem Mittelmaß“ ermöglichten, sich zum Nationalhelden und Diktator zu inszenieren. Ein ähnlicher Clown erhielt vor kurzem mehr als 70 Millionen Stimmen von amerikanischen Wählern, deren stagnierende Einkommen und begrenzte Bildungszugänge Piketty hervorragend dokumentiert. Von ihren traditionellen Unterstützern in der Demokratischen Partei als Bedauernswerte (Hillary Clinton's „deplorables“) abgetan, von immer sektiererischeren identitären Sozialwissenschaften ignoriert und von städtischen ,Eliten“ als Protofaschisten verdächtigt, wurden sie ein leichtes Opfer rechter Demagogik. In heutigen Gesellschaften kommt die ideologische Konsolidierung der Ungleichheit nur noch selten erkennbar von oben. An ihre Stelle tritt immer häufiger die opportunistische Ausnutzung von Umständen, die die Gewinner von Ungleichheit nicht selbst schufen, deren scheindemokratische Unterstützung sich jedoch als vorteilhaft für die Vermarktung ihrer Produkte und die Stärkung ihrer sozialen Position erweist.

Diesen Zusammenhang von Kapital und Ideologie berührt Piketty nur sehr gelegentlich in den letzten Kapiteln des Buches. Was hier fehlt, ist nicht nur ein umfassendes Verständnis von Prozessen, die bestehende Ungleichheitsstrukturen stabilisieren. Es geht auch um das Abschätzen von Möglichkeiten, Ungleichheit zu ändern. Piketty macht im letzten Teil seines Buches gut durchdachte Vorschläge für strukturelle Verteilungspolitiken, die dieses Ziel erreichen könnten. Das ist ein wichtiger Schritt. Wir müssen uns darüber hinaus aber auch Gedanken machen, wie wir unsere Mitbürger überzeugen können, solche Vorschläge zu akzeptieren. Marx argumentierte, dass die psychologischen Voraussetzungen für Revolutionen dann entstehen, wenn die meisten Betroffenen erkennen, dass die Realisierung wünschenswerter, notwendiger und machbarer gesellschaftlicher Ziele durch bestehende Ungleichheitsstrukturen blockiert wird. Solche Widersprüche zeigen sich heute vor allem im Umfeld eines potentiell fatalen globalen Klimawandels und einer immer grotesker werdenden Ungleichheit. In Toronto bot vor einigen Wochen ein Modehaus eine grüne Hutschachtel (ohne Hut) für 47,000 CAD (etwa 30,500€) an, ein bescheidenes Beispiel für die dreistelligen Millionen- 
beträge, die die Reichsten dieser Welt für Yachten oder Gemälde ausgeben, ohne sich sorgen zu müssen, ihre Konten in karibischen Steuerparadiesen übermäßig zu belasten.

Warum führt solch uferlose Ungleichheit nicht zu breitem öffentlichem Ärger und Widerstand? Warum brachen die 99\%-Proteste oder Fridays for Future, die Hunderttausende von Menschen auf die Straße brachten, so schnell wieder zusammen? Hier ist eine Ideologiekritik nötig, die nicht nur die Intentionen herrschender Gruppen, sondern auch die Vorstellungen und Lebensprobleme von Menschen untersucht, die Ungleichheit aus ihrer eigenen Perspektive sehen. Mehr noch geht es darum, zu verstehen, wie diese unterschiedlichen Reaktionen zur Verfestigung, aber auch zum Abbau von Ungleichheit beitragen können. An Studien dazu fehlt es nicht, vom Selbstbild des mittelalterlichen Bauerntums (Freedman, 1999) zum Verständnis der Sklaverei aus der Sicht von Sklaven (Baldus, 1977), vom Aufstieg selbstbesessener kultureller Eliten (Lasch, 1995) zum Konservatismus vormals progressiver Farmer und Arbeiter (Frank, 2004), vom ideologischen Einfluss moderner sozialer Medien (Benkler/Faris/Roberts, 2018) zum Zerfall von Gemeinschaft und Solidarität (Putnam/Garrett, 2020).

Vielleicht könnte man Piketty zu einem dritten Buch ermutigen, das diese so wichtigen Seiten der Ideologie untersucht. „Kapital im 21 Jahrhundert“ und „Ideologie und Kapital“ wären eine hervorragende Basis für ein solches Projekt.

\section{Literatur}

Baldus, B. Responses to Dependence in a Servile Group: The Machube of Northern Benin. In Slavery in Africa. Historical and Anthropological Perspectives; Miers, S.; Kopytoff, I., Eds.; University of Wisconsin Press: Madison, 1977; pp 435-458.

Baldus, B. Origins of Inequality in Human Societies; Routledge: London/New York, 2017. Benkler, Y.; Faris, R.; Roberts, H. Network Propaganda; Oxford University Press: New York, 2018. Frank, T. What's the Matter with Kansas?; Holt: New York, 2004.

Freedman, P. Images of the Medieval Peasant; Stanford University Press: Stanford 1999. Lasch, C. The Revolt of the Elites and the Betrayal of Democracy; W.W. Norton: New York, 1995. Putnam, R. D.; Garrett, S. R. The Upswing: How America Came Together a Century Ago and How We Can Do it Again; Simon \& Schuster: New York, 2020. 